\title{
ENABLING DELAY-SENSITIVE APPLICATIONS OVER SENSOR NETWORKS USING A TWO-TIER ARCHITECTURE AND MULTI-ANTENNA CLUSTER HEADS
}

\author{
L. Liu, J.-F. Chamberland \\ ECE Department \\ Texas A\&M University \\ College Station, TX 77843-3128, USA
}

\author{
K. Qaraqe \\ ECE Department \\ Texas A\&M University at Qatar \\ Doha, 23874, Qatar
}

\begin{abstract}
The timely processing and dissemination of information over wireless sensor networks is a key aspect of their future success. This is especially important for delay-sensitive applications such as detection and estimation. This paper assumes a two-tier architecture for wireless sensor networks where sensor nodes are simple devices equipped with one antenna, and cluster heads are more powerful units endowed with multiple antennas each. The cluster heads collect information from the sensor nodes, process data locally, and then relay pertinent information across the network. This article studies the potential benefits of having multiple antennas at the cluster heads on the data collection and dissemination operations. The focus is on delay-sensitive traffic, and performance is measured in terms of effective capacity. Analysis shows that, for Gaussian channels, having multiple antennas at the receiver results in a power gain, while having multiple antennas at the transmitter provides a statistical gain. In both scenarios, the data rate that a wireless channel can support is increased significantly through the use of multiple antennas at the cluster head. Numerical results confirm that the gains of a multipleantenna configuration over a single-antenna link are substantial for delay-sensitive applications. This provides support for the two-tier architecture studied in this paper and for cluster heads having multiple antennas.
\end{abstract}

\section{INTRODUCTION}

Our work is inspired by the vision that future wireless sensor networks may feature a two-tier architecture. The envisioned networks consist of a more capable wireless core coupled to a wide array of wireless sensors that gather information locally. Sensor nodes form small-scale clusters where data packets are transferred between nodes and, ultimately, to a nearby cluster head. The cluster heads are then responsible for processing the data and for further disseminating relevant information across the network. In our model, sensor nodes are simple

This work was supported in part by the Qatar Foundation for Education, Science, and Community Development, and by Qatar Telecom (Qtel), Qatar. This paper is dedicated to the memory of Sergio Servetto. devices with one antenna each, whereas the cluster heads are more powerful units that can be equipped with multiple antennas.

Wireless sensor networks, when used in the context of inference problems, are subject to stringent delay constraints. It is therefore important to design communication schemes that reduce congestion and delay in the network. In particular, delay restrictions imposed on a sensor network may preclude the use of long error-correcting codes, thereby forcing the system to operate away from its Shannon limit. Several notable contributions on the delay/throughput tradeoff in wireless systems have improved our understanding of delaysensitive communications $[1,2,3]$. Still, the literature on this topic is not fully developed; some aspects of delay-sensitive communications demand further investigation.

Herein, we study the interplay between the physical layer infrastructure and the queueing behavior of a wireless sensor network. Due to the time-varying nature of wireless channels, it is typically impossible to provide deterministic delay guarantees for specific links. Accordingly, we use a popular queueing metric for performance assessment. This metric captures the asymptotic decay-rate of buffer occupancy,

$$
\theta=-\lim _{x \rightarrow \infty} \frac{\log \operatorname{Pr}\{L>x\}}{x}
$$

where $L$ denotes the queue-length distribution of the buffer present at the transmitter. The parameter $\theta$ reflects the perceived quality of a communication link. A larger $\theta$ represents a better connection for delay-sensitive applications. This performance metric is closely related to the concepts of effective bandwidth [4] and effective capacity [5].

It is well-known that the use of antenna arrays can significantly increase the diversity [6] or the Shannon capacity [7] of wireless connections. We seek to identify the potential benefits of a multiple-antenna configuration for delay-sensitive traffic over sensor networks. Specifically, we are interested in the situation where only one of the wireless agents (namely the cluster head) is equipped with an antenna array. To insure that the sent information is received correctly at the destination, we assume that a feedback mechanism between the sensors and their respective cluster heads provides support for 
acknowledgments. Expressions for the effective capacities of single-antenna and vector Gaussian channels are derived under a block fading model. Our results suggest that there are substantial benefits in using multiple antennas at the cluster heads for delay-sensitive applications. At low SNRs, just as there is a power gain associated with using multiple receive antennas in terms of ergodic capacity [8], there is a statistical gain associated with using multiple transmit antennas in terms of effective capacity. This suggests that antenna arrays are especially suitable for delay constrained communication and hence wireless sensor networks.

\section{SYSTEM AND PERFORMANCE}

The effective capacity denotes the maximum constant arrival rate a system can support subject to a buffer-occupancy requirement specified by $\theta_{0}$. Consider the situation where the wireless channel is subject to block fading. That is, the channel coefficients stay invariant within a block of duration $T$, but vary independently from block to block. Let $r$ be a random variable that represents the system throughput during one block, the effective capacity of the service process is defined by

$$
\alpha(\theta)=-(1 / \theta T) \log E\left[e^{-\theta r}\right] .
$$

If the arrival rate $a$ satisfies $a \leq \alpha\left(\theta_{0}\right)$ then the exponent $\theta$ defined in (1) satisfies $\theta \geq \theta_{0}$ [4]. This characterization is tight; the decay requirement $\theta_{0}$ is satisfied if and only if $a \leq \alpha\left(\theta_{0}\right)$

\section{Fact 1. The function $\alpha(\theta)$ is monotonically decreasing.}

Fact 1 implies that the maximum admissible arrival rate decreases as the service requirement $\theta_{0}$ becomes more stringent. This reveals a fundamental tradeoff between system throughput and queueing performance.

Fact 2. The effective capacity is upper bounded by $E[r] / T$. Also, if $r \geq \kappa T$ almost surely for some constant $\kappa$, then $\alpha(\theta) \geq \kappa$.

Fact 2 provides two nontrivial bounds for the effective capacity of a wireless system. Specifically, the effective capacity is upper bounded by the ergodic capacity and lower bounded by the minimum instantaneous service rate of the underlying wireless channel.

We now study the effective capacities of single-antenna and multiple-antenna channels under Rayleigh block fading. Suppose that the wireless sensor node (or cluster head) has a mean power constraint $P$ and a total spectral bandwidth allocation $W$. Also, a large buffer is available at each wireless agent, where outgoing packets are stored before being transmitted to their destination. We assume that a transmitter sends uncorrelated circularly symmetric zero-mean complex Gaussian signals of equal power across all the transmit antennas [7]. Practical implementations with near optimal rates can be designed if the receiver has the ability to acknowledge reception of the data to ensure that the erroneous data is retransmitted $[9,10]$.

We denote the number of transmit antennas by $n_{T}$ and the number of receive antennas by $n_{R}$. In the case where a sensor node is transmitting to its cluster head, $n_{T}=1$. Conversely, when the cluster head sends data to a sensor node, $n_{R}=1$. Let $\mathbf{x}$ denote the $n_{T} \times 1$ vector of transmitted symbols, and $\mathbf{y}$ be the $n_{R} \times 1$ vector of received signals. Then,

$$
\mathbf{y}=\mathbf{H x}+\mathbf{n},
$$

where $\mathbf{H}$ is an $n_{R} \times n_{T}$ complex matrix, and $\mathbf{n}$ is an $n_{R} \times 1$ vector of additive white Gaussian noise. The element $h_{i, j}$ of $\mathbf{H}$ denotes the channel gain from transmit antenna $j$ to receive antenna $i$. We assume that the $\left\{h_{i, j}\right\}$ 's are independent and identically distributed (i.i.d.) zero-mean complex Gaussian random variables with unit variance. For a fixed $\mathbf{H}$, the mutual information between $\mathbf{x}$ and $\mathbf{y}$ is given by [7]

$$
I(\mathbf{H}, P)=W T \log \left(1+\|\mathbf{H}\|^{2} P / n_{T} N_{0} W\right)
$$

where $\|\mathbf{H}\|^{2}$ denotes the $l_{2}$ norm of the channel vector $\mathbf{H}$.

\subsection{Single-Antenna System}

In the situation where the service process is governed by a single-input single-output (SISO) channel, the channel vector $\mathbf{H}$ reduces to a scalar $h$ for every block. The channel capacity $r$ during each block can be expressed as

$$
r=W T \log \left(1+|h|^{2} P / N_{0} W\right) \text { nats per second, }
$$

where $N_{0} / 2$ denotes the power spectral density of the noise process. The moment generating function of the service process $E\left[e^{-\theta r}\right]$ can be written as follows,

$$
e^{\frac{N_{0} W}{P}}\left(P / N_{0} W\right)^{-\theta W T} \Gamma\left(1-\theta W T, N_{0} W / P\right)
$$

where $\Gamma(z, x)$ is the upper incomplete gamma function. The effective capacity for the SISO system can be obtained as

$$
\begin{aligned}
\alpha(\theta) & =W \log \left(P / N_{0} W\right) \\
& -(1 / \theta T)\left(\log \left(\Gamma\left(1-\theta W T, N_{0} W / P\right)\right)+N_{0} W / P\right) .
\end{aligned}
$$

In the low SNR regime, as is the case with sensor networks, the nature of the effective capacity for the SISO system can be seen more clearly. At low SNRs, the approximation $\log (1+$ $s) \approx s$ holds and the effective capacity can be expressed as

$$
\alpha(\theta)=(1 / \theta T) \log \left(1+\theta T P / N_{0}\right) .
$$

When the mean received power $P$ is small, the system is operating in the power-limited regime and this first-order approximation is very accurate. To further our understanding of the SISO system, we analyze (4) in both the delay-insensitive regime and the delay-intolerant asymptotics. In the low SNR 
regime, the effective capacity converges to the ergodic capacity of the wireless system as $\theta \downarrow 0$,

$$
\lim _{\theta \downarrow 0} \alpha(\theta)=P / N_{0} \approx E\left[W \log \left(1+|h|^{2} P / N_{0} W\right)\right] .
$$

As $\theta \uparrow \infty$, the effective capacity of the SISO system vanishes, $\lim _{\theta \uparrow \infty} \alpha(\theta)=0$. Thus, this sensor network cannot support delay-intolerant traffic. For any arrival rate $\epsilon>0$, there exits a non-negligible probability that $\epsilon$ exceeds the instantaneous system capacity $r$ in (3). These findings are hardly surprising in the light of Fact 2.

Define the first-order derivative of the effective capacity as the decay function of the wireless link,

$$
\alpha^{\prime}(\theta)=\frac{\theta T^{2} P-\left(N_{0} T+\theta T^{2} P\right) \log \left(1+\frac{\theta T P}{N_{0}}\right)}{\theta^{2} T^{2}\left(N_{0}+\theta T P\right)} .
$$

The magnitude of this function indicates the decay speed of $\alpha(\theta)$ as $\theta$ increases. Let $\rho \triangleq-\lim _{\theta \downarrow 0} \alpha^{\prime}(\theta)$, denote the initial decay-rate of the effective capacity for the corresponding wireless system. We note that the initial decay-rate for the SISO system (which will soon be useful) is equal to

$$
\rho_{\text {SISO }}=T P^{2} / 2 N_{0}^{2} \text {. }
$$

\subsection{Multiple-Antenna Systems}

Next, we explore the more general situation where the service process of a wireless system is governed by a multipleantenna channel. The effective capacity for such channels is obtained based on the moment generating function of its mutual information. The performance gains of the vector Gaussian systems over the SISO configuration are evaluated in the low SNR regime. Our results suggest that, at low SNRs, a multiple receive antenna configuration provides a power gain of $n_{R}$ and a multiple transmit antenna configuration provides a statistical gain of $n_{T}$ over a single-antenna system.

Consider the situation where the wireless link has one transmit antenna and $n_{R}$ receive antennas. The channel matrix of this single-input multiple-output (SIMO) system $\mathbf{H}=$ $\left[h_{1,1}, \ldots, h_{n_{R}, 1}\right]^{T}$ becomes an $n_{R} \times 1$ vector of i.i.d. complex Gaussian random variables. During each block, the realized system throughput can be expressed as

$$
r=W T \log \left(1+\sum_{k=1}^{n_{R}}\left|h_{k, 1}\right|^{2} \frac{P}{N_{0} W}\right) \quad \text { nats per second. }
$$

Using the low SNR approximation and the fact that the components of the channel vector $\mathbf{H}$ are independent, $E\left[e^{-\theta r}\right]$ can be expressed as

$$
\left[\int_{0}^{\infty} e^{-\frac{\theta T|h|^{2} P}{N_{0}}} e^{-|h|^{2}} d|h|^{2}\right]^{n_{R}}=\left(1+\theta T P / N_{0}\right)^{-n_{R}} .
$$

Accordingly, the effective capacity of the SIMO system in the low SNR regime is found to be

$$
\alpha(\theta)=\left(n_{R} / \theta T\right) \log \left(1+\theta T P / N_{0}\right) .
$$

Comparing (6) with (4), we find that the use of multiple receive antenna results in a power gain of $n_{R}$.

The effective capacity and the low SNR approximation of this SIMO system appear in Fig. 1 for $P=10 \mathrm{~mW}, W=1$ MHz, $T=5 \mathrm{~ms}$, and $N_{0}=10^{-6} \mathrm{~W} / \mathrm{Hz}$. We note that the

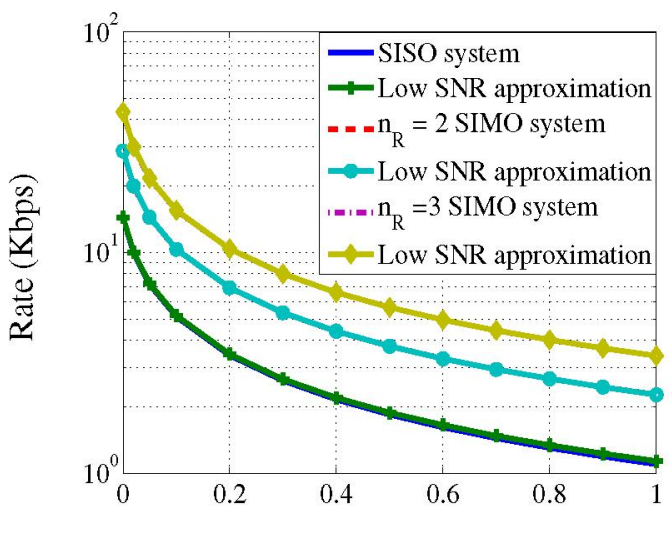

Exponential Requirement $\theta_{0}$

Fig. 1. Effective capacity for SIMO systems.

approximation of the effective capacity for the SIMO system in the low SNR regime is quite accurate. Furthermore, the effective capacity of the SIMO system scales linearly with the number of receive antennas for all service constraint $\theta_{0}$. This result can be viewed as an extension of the statement that the ergodic capacity of the SIMO system scales linearly with $n_{R}$ in the low SNR regime [8].

In the situation where the service process is governed by a multiple-input single-output (MISO) channel ( $n_{T}$ transmit antennas and one receive antenna), the channel matrix $\mathbf{H}=$ $\left[h_{1,1}, \ldots, h_{1, n_{T}}\right]$ becomes a $1 \times n_{T}$ vector of i.i.d. complex Gaussian random variables. The realized system throughput of the MISO channel during each block can be expressed as

$$
r=W T \log \left(1+\sum_{k=1}^{n_{T}}\left|h_{1, k}\right|^{2} \frac{P}{N_{0} W n_{T}}\right)
$$

Using the low SNR approximation and the independence assumption between the components of $\mathbf{H}, E\left[e^{-\theta r}\right]$ simplifies to

$$
\left[\int_{0}^{\infty} e^{-\frac{\theta T|h|^{2} P}{N_{0} n_{T}}} e^{-|h|^{2}} d|h|^{2}\right]^{n_{T}}=\left(1+\theta T P / N_{0} n_{T}\right)^{-n_{T}} .
$$

The effective capacity of the service process for the MISO system can then be expressed as

$$
\alpha(\theta)=\left(n_{T} / \theta T\right) \log \left(1+\theta T P / N_{0} n_{T}\right) .
$$

The gain of using multiple transmit antennas over the singleantenna case is not entirely obvious by looking at (4) and (7). As such, we compare the values of the effective capacity in 
two asymptotic regimes for the service requirements. We know that, without power allocation, having multiple transmit antennas does not increase the ergodic capacity at low SNRs [8]. This can also be seen by evaluating the effective capacity as $\theta \downarrow 0$,

$$
\lim _{\theta \downarrow 0} \alpha(\theta)=\frac{P}{N_{0}} \approx E\left[W \log \left(1+\sum_{i=1}^{n_{T}}\left|h_{i}\right|^{2} \frac{P}{N_{0} W n_{T}}\right)\right] .
$$

However, there is a statistical gain of $n_{T}$ associated with a multiple transmit antenna configuration. This statistical gain is seen more clearly from the decay function of the effective capacity. The decay function of the MISO system is given by

$$
\alpha^{\prime}(\theta)=\frac{n_{T} \theta T P-\left(N_{0} n_{T}^{2}+n_{T} \theta T P\right) \log \left(1+\frac{\theta T P}{N_{0} n_{T}}\right)}{\theta^{2} T\left(N_{0} n_{T}+\theta T P\right)},
$$

and the initial decay-rate of the corresponding effective capacity becomes

$$
\rho_{\text {MISO }}=T P^{2} / 2 n_{T} N_{0}^{2} .
$$

Comparing (5) and (8), we find that the decay-rate of the effective capacity for the MISO system is $n_{T}^{-1}$ times that of the single-antenna system. This implies that having multiple transmit antennas reduces the decay of the effective capacity as a function of $\theta$. This is especially beneficial for delay sensitive traffic. Since both the SIMO system and the SISO system have the same ergodic capacity in the low SNR regime, we define the statistical gain of the MISO system over the singleantenna system by

$$
g=\rho_{\text {SISO }} / \rho_{\text {MISO }}=n_{T} .
$$

The multiple transmit antenna configuration results in a statistical gain of $n_{T}$, but no gain in terms of ergodic capacity. It is also interesting to note that as $n_{T} \uparrow \infty, \rho_{\text {MISO }} \downarrow 0$ which means that as the number of transmit antenna becomes large the effective capacity does not decay at all when the exponential requirement $\theta$ is small. For the same parameters as in the SIMO case, the exact and approximated effective capacities for the SISO and MISO systems are shown in Fig. 2. The ergodic capacities of the $2 \times 1$ system and the $3 \times 1$ system are the same as that of the single-antenna system. Using multiple transmit antennas induces a statistical gain that prevents the effective capacity from decaying rapidly as a function of $\theta_{0}$. Furthermore, as the service requirement $\theta$ increases, the gains in terms of the effective capacity for the MISO systems over the SISO system become larger.

\section{CONCLUSION}

We studied the benefits of a multiple-antenna configuration on delay-sensitive traffic over wireless sensor networks. The effective capacities of the single-antenna and vector-Gaussian

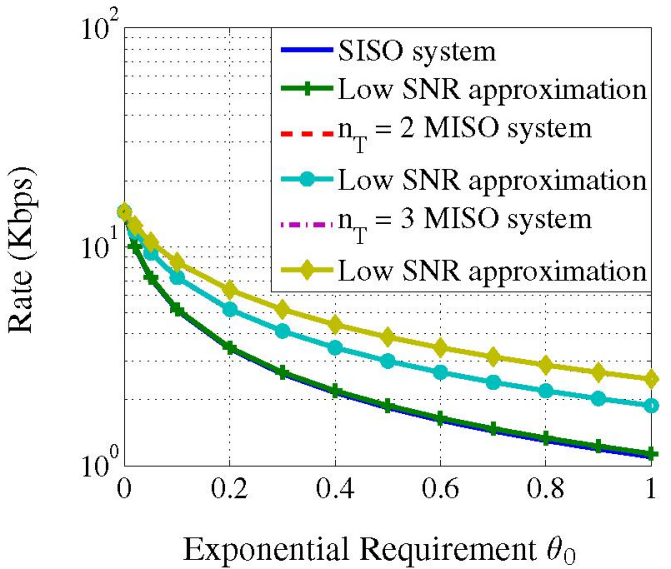

Fig. 2. Effective capacity for MISO systems.

systems were characterized. Our results demonstrate a clear tradeoff between throughput and service quality. In the low SNR regime, having multiple transmit antennas can provide a statistical gain of $n_{T}$, while multiple receive antennas result in a power gain of $n_{R}$. This result suggests that multiple-antenna configurations are especially helpful for delay-sensitive communication over wireless sensor networks.

\section{REFERENCES}

[1] A. Ephremides and B. Hajek, "Information theory and communication networks: An unconsummated union," IEEE Trans. Inf. Theory, vol. 44, no. 6, pp. 2416 - 2434, October 1998.

[2] R. Berry and R. Gallagar, "Communication over fading channels with delay constraints," IEEE Trans. Inf. Theory, vol. 48, no. 5, pp. 1135 - 1149, May 2002.

[3] M. Neely and E. Modiano, "Capacity and delay tradeoffs for ad-hoc mobile networks," IEEE Trans. Inf. Theory, vol. 51, no. 6, pp. 1917 - 1937, June 2005.

[4] C.-S. Chang, Performance Guarantees in Communication Networks. Springer-Verlag, 2000.

[5] D. Wu and R. Negi, "Effective capacity: a wireless link model for support of quality of service," IEEE Trans. Wireless Commun., vol. 2, no. 4, pp. 630-643, July 2003.

[6] V. Tarokh, H. Jafarkhani, and A. R. Calderbank, "Space-time block code from orthogonal designs," IEEE Trans. Inf. Theory, vol. 45, no. 5, pp. 1456-1467, July 1999.

[7] E. Telatar, "Capacity of multi-antenna gaussian channels," European Transactions on Telecommunication, vol. 10, no. 6, pp. 585-596, November 1999.

[8] D. Tse and P. Viswanath, Fundamentals of Wireless Communication. Cambridge University Press, 2005.

[9] M. Luby, "LT codes," in Forty-Third Annual IEEE Symposium on Foundations of Computer Science, 2002, pp. $271-280$.

[10] A. Shokrollahi, "Raptor codes," in IEEE International Symposium on Information Theory, 2004. 